\title{
Shikonin sensitizes wild-type EGFR NSCLC cells to erlotinib and gefitinib therapy
}

\author{
YANG-LING LI ${ }^{1}$, XIU HU ${ }^{2,3}$, QING-YU LI ${ }^{1}$, FEI WANG ${ }^{1}$, BO ZHANG $^{4}$, KE DING $^{1}$, \\ BI-QIN TAN $^{1}$, NENG-MING LIN ${ }^{1,4}$ and CHONG ZHANG ${ }^{2}$
}

\begin{abstract}
${ }^{1}$ Department of Clinical Pharmacology, Affiliated Hangzhou First People's Hospital, Zhejiang University School of Medicine, Hangzhou, Zhejiang 310006; ${ }^{2}$ School of Medicine, Zhejiang University City College, Hangzhou, Zhejiang 310015;

${ }^{3}$ College of Pharmaceutical Sciences, Zhejiang University, Hangzhou, Zhejiang 310058;

${ }^{4}$ Hangzhou Translational Medicine Research Center, Affiliated Hangzhou First People's Hospital,

Zhejiang University School of Medicine, Hangzhou, Zhejiang 310006, P.R. China
\end{abstract}

Received April 9, 2018; Accepted July 19, 2018

DOI: $10.3892 / \mathrm{mmr} .2018 .9347$

\begin{abstract}
As patients with non-small cell lung cancer (NSCLC) and wild-type epidermal growth factor receptor (EGFR) are resistant to treatment with erlotinib or gefitinib, potential chemosensitizers are required to potentiate wild-type EGFR NSCLC cells to erlotinib/gefitinib treatment. The present study reported that shikonin could sensitize the anticancer activity of erlotinib/gefitinib in wild-type EGFR NSCLC cells. Furthermore, shikonin could potentiate mitochondrial-mediated apoptosis induced by erlotinib/gefitinib in wild-type EGFR NSCLC cells. In addition, the present study demonstrated that shikonin could induce apoptosis by activating reactive oxygen species (ROS)-mediated endoplasmic reticulum (ER) stress, and that erlotinib/gefitinib may also induce ER stress in wild-type EGFR NSCLC cells; however, shikonin plus erlotinib/gefitinib was more effective in activating ER stress than either agent alone. This indicated that ROS-mediated ER stress may be associated with enhanced mitochondrial apoptosis induced by shikonin plus erlotinib/gefitinib. In addition, shikonin may promote the transition of cytoprotective ER stress-inducing EGFR-tyrosine kinase inhibitor tolerance to apoptosis-promoting ER stress. Furthermore, shikonin may enhance the anti-NSCLC activity of erlotinib/gefitinib in vivo. The data of the present study indicated that shikonin may be a potential sensitizer to enhance
\end{abstract}

Correspondence to: Dr Neng-Ming Lin, Department of Clinical Pharmacology, Affiliated Hangzhou First People's Hospital, Zhejiang University School of Medicine, 261 Huansha Road, Hangzhou, Zhejiang 310006, P.R. China

E-mail:1nm1013@163.com

Dr Chong Zhang, School of Medicine, Zhejiang University City College, 51 Huzhou Street, Hangzhou, Zhejiang 310015, P.R. China

E-mail: zhangchong@zucc.edu.cn

Key words: shikonin, erlotinib, gefitinb, combination, ROS-ER stress, EGFR-TKIs the anti-cancer efficacy of erlotinib/gefitinib in wild-type EGFR NSCLC cells resistant to erlotinib/gefitinib treatment.

\section{Introduction}

Globally, lung cancer is the second most common newly diagnosed cancer and the leading cause of cancer-associated mortality. Non-small cell lung cancer (NSCLC) accounts for $\sim 83 \%$ of all newly diagnosed lung cancers (1). Numerous patients with NSCLC are diagnosed at an advanced stage, thus surgery may not be suitable; chemotherapy is a major option to treat such patients (2). First-generation epidermal growth factor receptor-tyrosine kinase inhibitors (EGFR-TKIs), including erlotinib and gefitinib have demonstrated notable response rates and benefits in progression-free survival compared with first-line conventional platinum-based chemotherapy (3). However, EGFR-TKIs have become first-line therapy drugs for patients with NSCLC harboring EGFR-activating mutations; the majority of patients with an initial response to erlotinib or gefitinib will eventually develop resistance due to the effects of drug-resistant mutations, with T790M mutations accounting for $\sim 60 \%$ of all resistance $(4,5)$. Additionally, patients with NSCLC and wild-type EGFR are primarily resistant to erlotinib and gefitinib (6,7). Thus, potential chemosensitizers are required to sensitize wild-type EGFR NSCLC cells to EGFR-TKI treatment.

Shikonin, a natural naphthoquinone derivative isolated from the traditional medical herb Lithospermum erythrorhizon, has antitumor, anti-inflammatory, antimicrobial, and antithrombotic properties (8). Shikonin has been reported to exhibit antitumor properties via the generation of reactive oxygen species (ROS), activating endoplasmic reticulum (ER) stress, inducing caspase-dependent apoptosis and inhibiting angiogenesis (9-12). Furthermore, shikonin can sensitize cancer cells to numerous types of treatment with cytotoxic drugs $(11,13,14)$. In addition, shikonin can also suppress EGFR signaling and enhance the anti-glioblastoma efficacy of erlotinib via inhibiting EGFR in vitro (15). Shikonin inhibits gefitinib-resistant NSCLC cells by inhibiting thioredoxin reductase and activating the EGFR proteasomal degradation 
pathway in vitro (16); however, whether shikonin can enhance the anticancer activity of erlotinib or gefitinib in wild-type EGFR NSCLC cells requires further investigation.

In the present study, the anticancer activity of combining shikonin plus erlotinib/gefitinib was evaluated in wild-type EGFR NSCLC cells in vitro and in vivo. In addition, the findings of the resent study demonstrated that shikonin could promote erlotinib/gefitinib-induced apoptosis in wild-type EGFR NSCLC cells. ER stress and ROS were associated with shikonin plus erlotinib/gefitinib-induced apoptosis. Thus, the present study proposed that shikonin may be a potential sensitizer to enhance the anticancer efficacy of erlotinib/gefitinib in wild-type EGFR NSCLC cells primarily resistant to erlotinib/gefitinib treatment.

\section{Materials and methods}

Materials. Shikonin (cat. no. S8279) and gefitinib (cat. no. S1025) were obtained from Selleck Chemicals (Houston, TX, USA). Erlotinib (cat. no. 183321-74-6) was obtained from LC Laboratories (Woburn, MA, USA). N-acetylcysteine (NAC; cat. no. A0737) was obtained from Sigma-Aldrich (Merck KGaA, Darmstadt, Germany).

Cell culture. A549 (cat. no. TCHu150), NCI-H1299 (cat. no. TCHu160), NCI-H460 (cat. no. TCHu205), 95-D (cat. no. TCHu 61) and NCI-H1650 (cat. no. TCHu152) cells were obtained from the Shanghai Institute of Biochemistry and Cell Biology (Chinese Academy of Sciences, Shanghai, China). NCI-H1650, NCI-H1299, NCI-H460 and 95-D cells were cultured in RPMI-1640 medium containing 10\% fetal bovine serum (FBS); A549 cells were maintained in Ham's F12 medium+10\% FBS.

Cell viability assay. NSCLC cells were treated with serial dilutions of gefitinib, erlotinib and shikonin for $72 \mathrm{~h}$. A sulforhodamine blue (SRB) assay was used to test the proliferation of cancer cells as described previously (17).

Determination of cell colony formation. NSCLC cells were plated at $\sim 700$ cells per dish. The next day, compounds (gefitinib, erlotinib, shikonin, shikonin+erlotinib and shikonin+ gefitinib) were added into the NSCLC cells; the medium containing $10 \%$ FBS plus the compounds was replaced every 3 days. Dishes were stained with $0.5 \%$ crystal violet after 14 days treatment. Finally, the colonies were scored and photographed.

Propidium iodide (PI) staining and determination of mitochondrial membrane depolarization. Cancer cells $\left(3 \times 10^{5} /\right.$ well) were exposed to the drugs, harvested and washed with PBS. Then, PI staining was used to detect apoptosis, and the mitochondrial membrane depolarization was determined by 5,5',6,6'tetrachloro-1,1',3,3'tetraethylbenzimidazol-carbocyanine iodide staining as described previously (17).

Western blot analysis. Western blotting was conducted as described previously (17). The primary antibodies below were used in the present study: Induced myeloid leukemia cell differentiation protein 1 (Mcl-1; cat. no. sc-819), poly (ADP-ribose) polymerase (PARP; cat. no. sc-7150) and caspase-3 (cat. no. sc-7148) were purchased from Santa Cruz Biotechnology, Inc. (Dallas, TX, USA). Antibodies for cleaved-caspase-3 (cat. no. 9661), activating transcription factor 4 (ATF-4; cat. no. 11815), phosphorylated (p)-eukaryotic initiation factor $2 \alpha$ (eIf $2 \alpha$; cat. no. 3398) and eIf $2 \alpha$ (cat. no. 5324) were purchased from Cell Signaling Technology, Inc. (Danvers, MA, USA). $\beta$-actin (cat. no. BD-612656) was obtained from BD Biosciences (Franklin Lakes, NJ, USA).

Animals and antitumor activity in vivo. Human lung cancer A549 xenografts were established as described previously (18). The nude mice were randomized to 6 groups and then treated with vehicle, shikonin $[20 \mathrm{mg} / \mathrm{kg}$, intraperitoneal (i.p.) administration] once per week, erlotinib $[50 \mathrm{mg} / \mathrm{kg}$, intragastric (i.g.) administration] twice per week, gefitinib $(50 \mathrm{mg} / \mathrm{kg}$, i.g. administration) twice per week, shikonin $(20 \mathrm{mg} / \mathrm{kg}$, i.p., once per week) + erlotinib (50 mg/kg, i.g., twice per week) and shikonin $(20 \mathrm{mg} / \mathrm{kg}$, i.p., once per week $)+$ gefitinib $(50 \mathrm{mg} / \mathrm{kg}$, i.g., twice per week) for 35 days. All animal protocols were performed in accordance with the National Institutes of Health Guide for the Care and Use of Laboratory Animals, and were approved by the Zhejiang University City College Animal Care and Use committee. This article does not contain any studies with human participants performed by any of the authors.

Statistical analysis. One-way analysis of variance followed with the Tukey's post hoc test was used to examine the significance of differences among groups, and two-tailed student's $t$ tests was used to examine the significance of differences two groups. Data points in graphs were presented as the mean \pm standard deviation. ${ }^{*} \mathrm{P}<0.05$ was considered to indicate a statistically significant difference, ${ }^{* *} \mathrm{P}<0.01$ and ${ }^{* * *} \mathrm{P}<0.001$ were considered to indicate a highly statistically significant difference. For the SRB assay, combination index (CI) values were calculated using Calcusyn (http://www.biosoft. com/w/calcusyn.htm) and the mean CI values were chosen for presentation (19). $\mathrm{CI}<0.9$ indicated synergism; 0.9 to 1.10 , additive and $>1.10$, antagonism.

\section{Results}

Shikonin sensitizes the antiproliferative effects of erlotinib/gefitinib in wild-type EGFR NSCLC cells. Firstly, antiproliferative effects were investigated by combining shikonin with erlotinib/gefitinib in vitro. As expected, shikonin could potentiate erlotinib/gefitinib-induced cytotoxicity in NSCLC cells (Fig. 1). CI values were calculated with Calcusyn software; treatment with shikonin plus erlotinib/gefitinib revealed synergistic cytotoxic effects in NSCLC cells, with the mean CI values below 0.9. In addition, the combined treatment of shikonin plus erlotinib/gefitinib was markedly more effective in attenuating colony formation than either agent alone (Fig. 2). Thus, shikonin plus erlotinib/gefitinib was effective in limiting colony formation and cell growth in wild-type EGFR NSCLC cells.

Shikonin promotes erlotinib/gefitinib-induced apoptosis. The present study also investigated whether shikonin could 

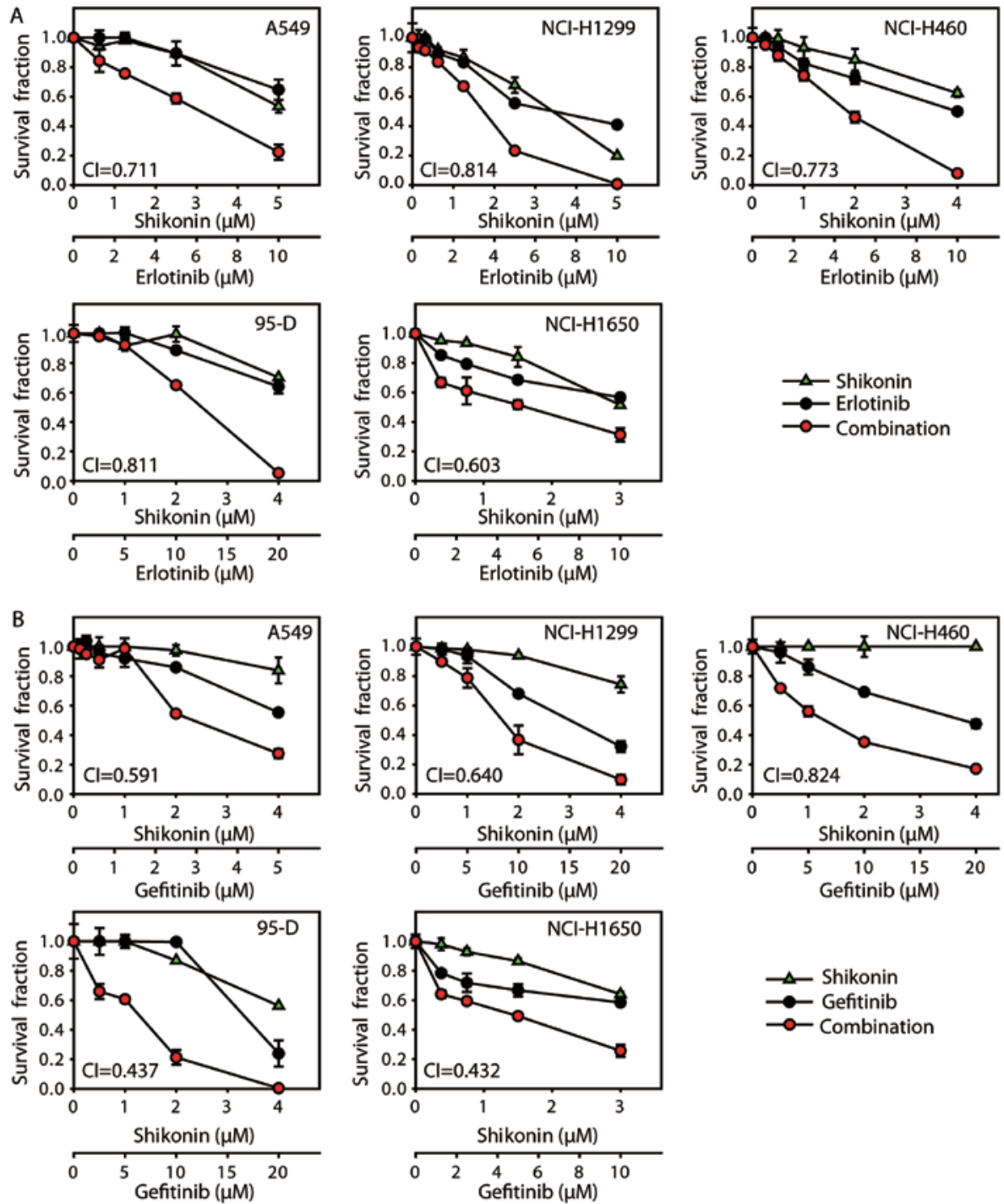

Figure 1. Shikonin sensitizes the anti-proliferation effects of erlotinib/gefitinib. (A) Cells were incubated with shikonin and/or erlotinib for $72 \mathrm{~h}$. (B) Cells were incubated with shikonin and/or gefitinib for $72 \mathrm{~h}$. SRB assay was used to detect the proliferation of cancer cells. SRB, sulforhodamine blue.

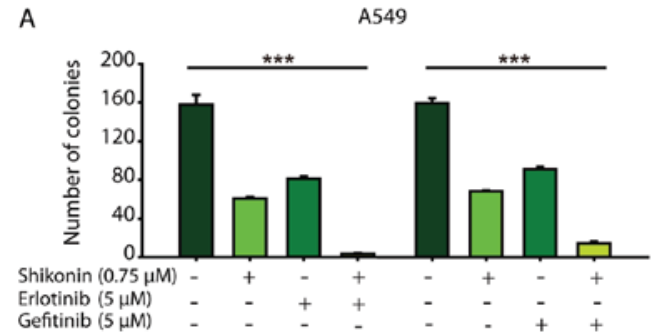

B

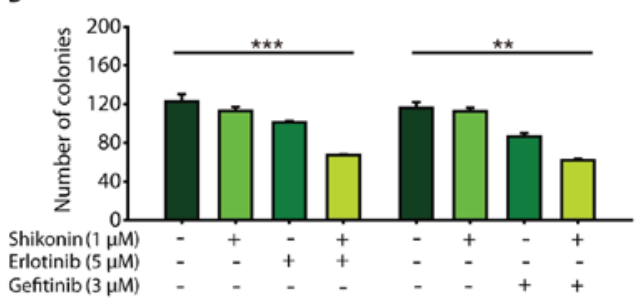

Figure 2. Shikonin sensitizes the anti-proliferation effects of erlotinib/gefitinib in colony formation assay. (A) A549 cells were incubated with shikonin plus erlotinib/gefitinib for 14 days, and cell colony formation assay was determined. (B) Shikonin sensitized the anti-proliferation effects of erlotinib/gefitinib in NCI-H1299 colony formation assay. ${ }^{* *} \mathrm{P}<0.01$ and ${ }^{* * * *} \mathrm{P}<0.001$. promote erlotinib/gefitinib-induced apoptosis in wild-type EGFR NSCLC cells; PI staining was used to detect apoptosis. Consistent with the cytotoxicity data, it was demonstrated that shikonin could promote erlotinib-induced apoptosis in NSCLC cells (Fig. 3A, upper panel and 3B). In addition, shikonin plus erlotinib resulted in greater mitochondrial membrane depolarization than either drug alone in wild-type EGFR NSCLC cells (Fig. 3A, lower panel and C). Furthermore, shikonin was revealed to potentiate mitochondrial-mediated apoptosis induced by gefitinib in wild-type EGFR NSCLC cells (Fig. 4). Additionally, shikonin plus erlotinib/gefitinib markedly induced cleavage of caspase- 3 and PARP, and inhibition of Mcl-1 in NSCLC cells (Fig. 5A). Collectively, the results of the present study suggested that shikonin could promote erlotinib/gefitinib-induced apoptosis via the mitochondrial apoptotic pathway in wild-type EGFR NSCLC cells.

ER stress and ROS are associated with shikonin plus erlotinib/gefitinib-induced apoptosis in vitro. Shikonin induced ROS generation, activated ER stress and induced 

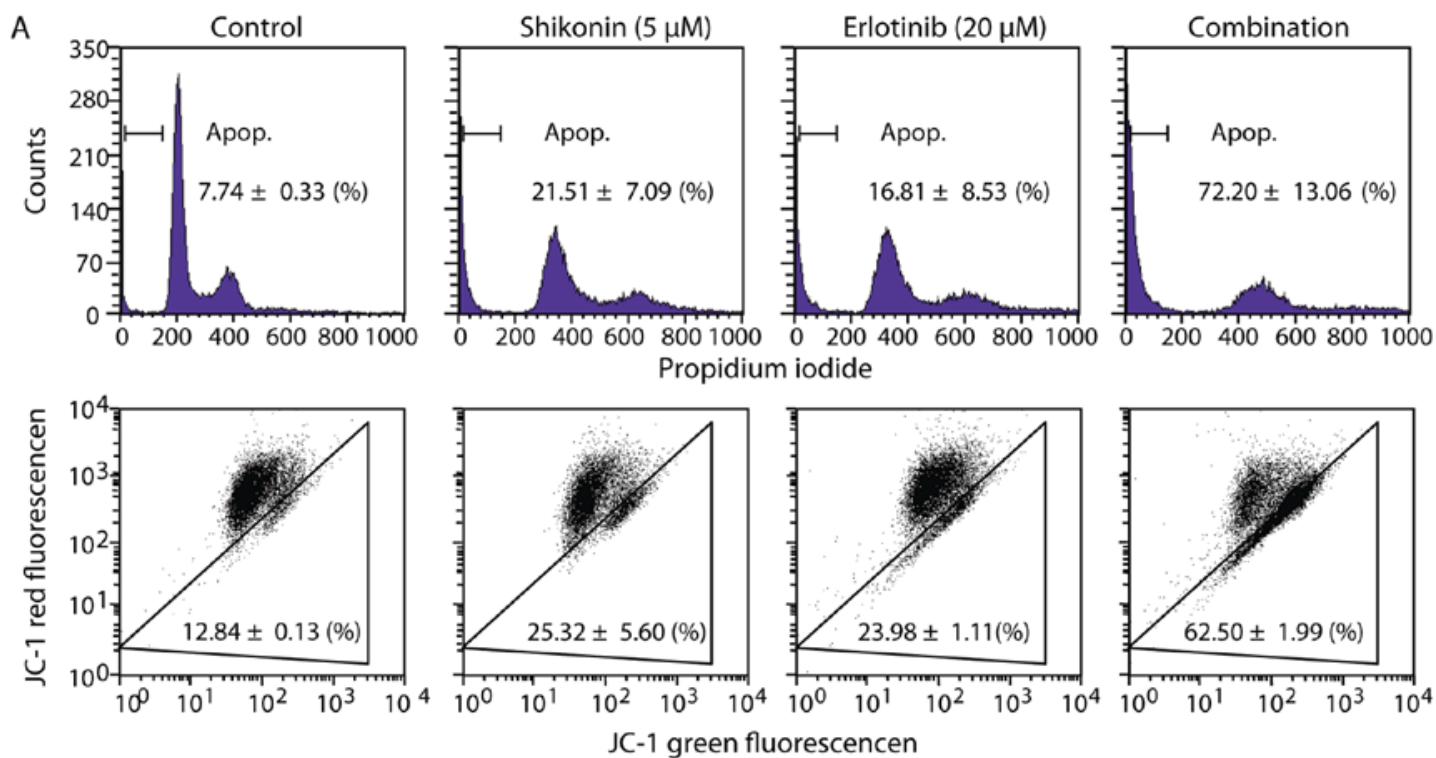

B
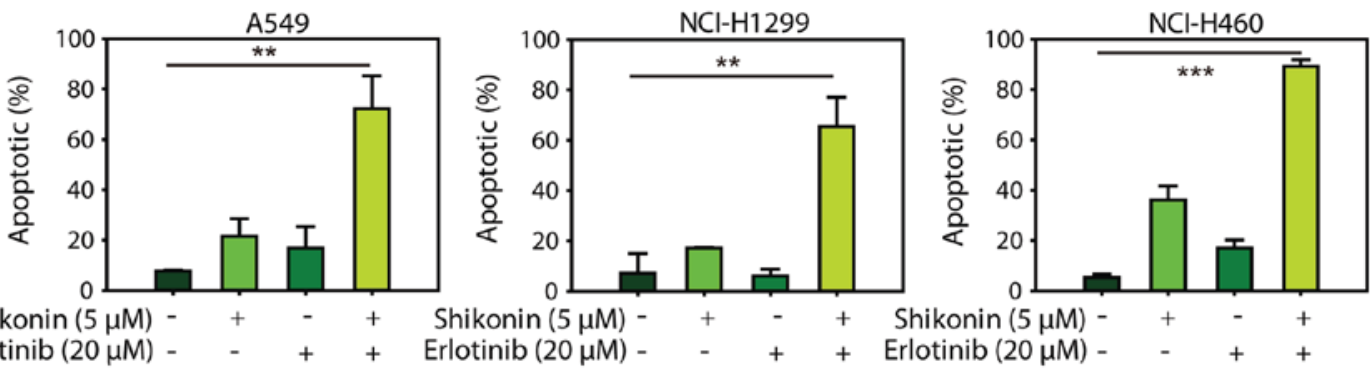

C
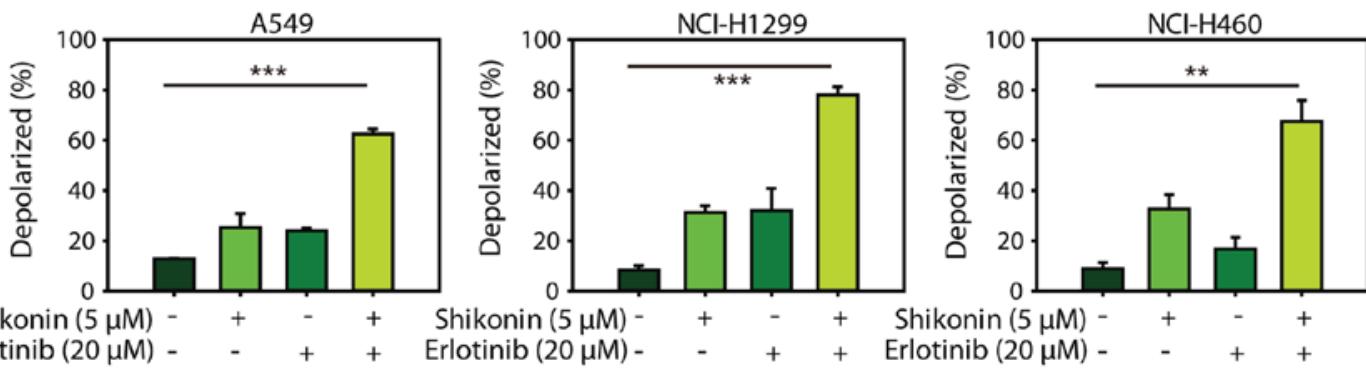

Figure 3. Shikonin promotes erlotinib-induced apoptosis. (A) A549 cells were treated with shikonin $(5 \mu \mathrm{M})$, erlotinib $(20 \mu \mathrm{M})$ or the combination for $48 \mathrm{~h}$, and then cells were incubated with PI (upper panel) or JC-1 (lower panel) and analyzed by flow cytometry. (B) Shikonin promoted erlotinib-induced apoptosis in A549, NCI-H1299 and NCI-H460 cells. (C) Shikonin promoted mitochondrial membrane potential induced by erlotinib in three NSCLC cells. ${ }^{* *} \mathrm{P}<0.01$ and ${ }^{* * *} \mathrm{P}<0.001$. NSCLC, non-small cell lung cancer; PI, propidium iodide.

mitochondrial-associated apoptosis in human prostate cancer cells (12). Therefore, the present study first examined whether shikonin could induce ROS-mediated ER stress in wild-type EGFR NSCLC cells. As presented in Fig. 5B, NAC was observed to reverse the apoptosis induced by shikonin treatment. Furthermore, NAC was demonstrated to also protect cells from apoptosis induced by shikonin plus erlotinib/gefitinib. These data indicated that ROS may be associated with the enhanced apoptosis induced by shikonin plus erlotinib/gefitinib. The present study also detected the expression levels of ER stress marker proteins (eIF2a and ATF4), which may be activated during ER stress. Shikonin increased the expression levels of p-eIF2a and ATF4 in the absence of erlotinib/gefitinib, and this effect was markedly enhanced in the presence of erlotinib/gefitinib (Fig. 5C). In addition, NAC pretreatment inhibited the activation of ATF-4 and eIF2a induced by shikonin plus erlotinib/gefitinib. Furthermore, the activation of caspase-3 induced by shikonin plus erlotinib/gefitinib was completely inhibited by NAC pretreatment (Fig. 5D). Thus, the data of the present study suggested that ROS-mediated ER stress was involved in shikonin plus erlotinib/gefitinib-induced apoptosis in wild-type EGFR NSCLC cells.

Shikonin sensitizes the anti-NSCLC effects of erlotinib/gefitinib in vivo. Finally, the in vivo activity of shikonin plus erlotinib/gefitinib was detected on A549 xenograft model. The dose of erlotinib/gefitinib selected in the present study was not expected to exhibit significant anticancer efficacy. As presented in Fig. 6, the low dose of erlotinib/gefitinib did not result in significant tumor growth inhibition; however, combining shikonin and erlotinib/gefitinib significantly inhibited tumor growth [mean Relative Tumor Volume (RTV) ${ }_{\text {shikonin }}$ 

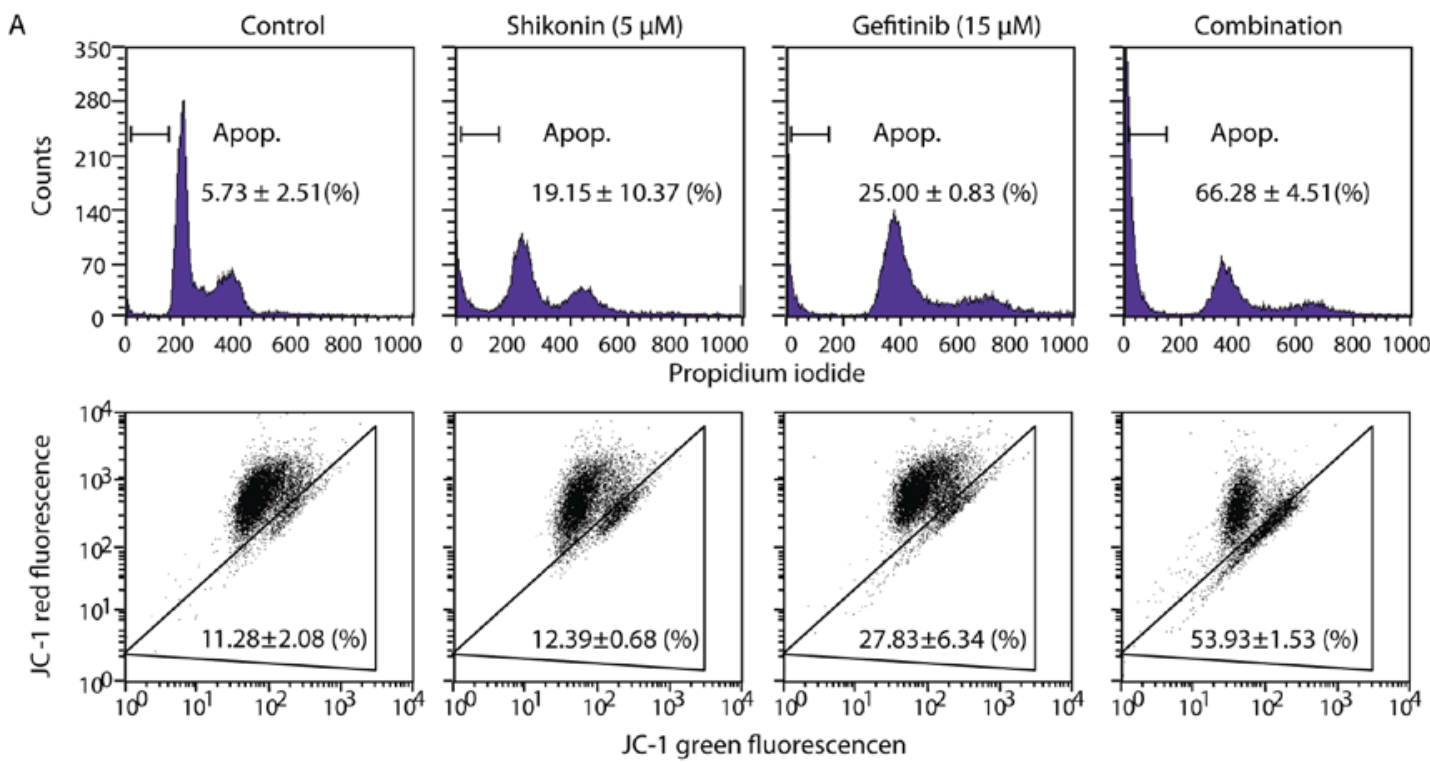

B
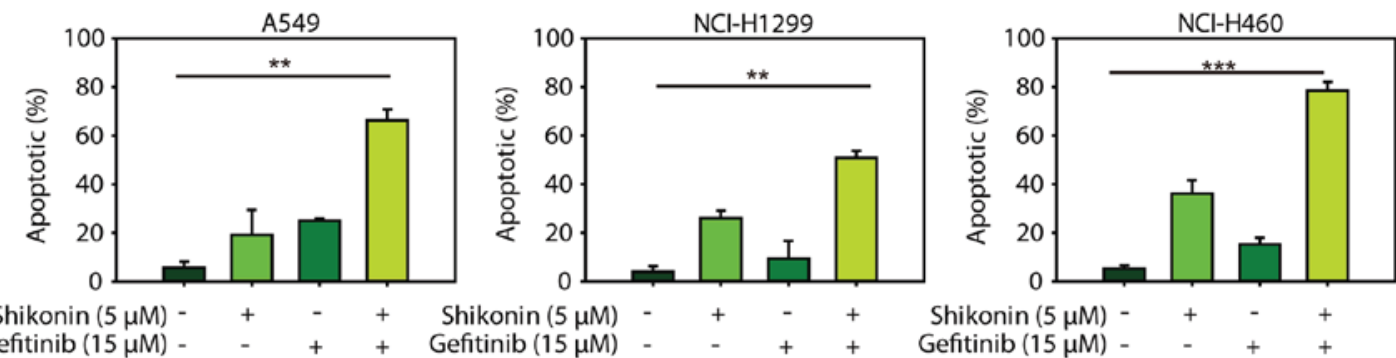

C
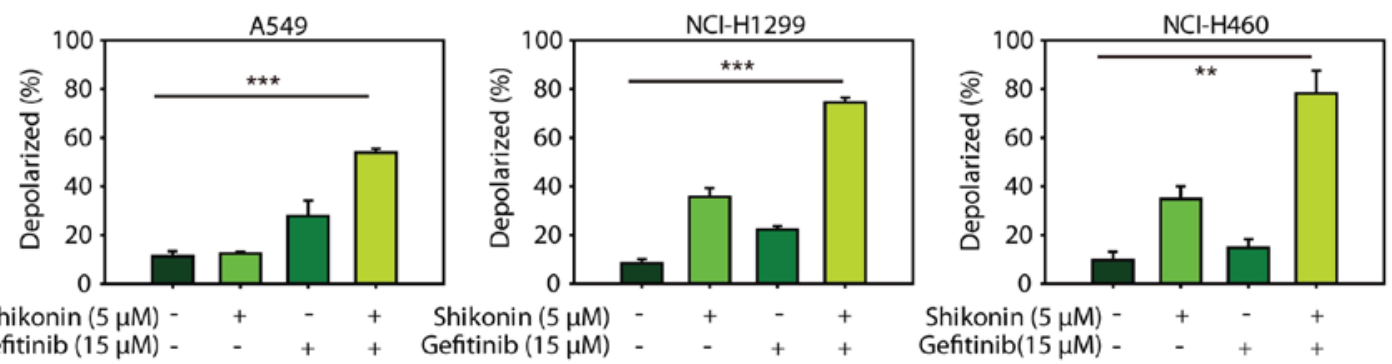

Figure 4. Shikonin promotes gefitinib-induced apoptosis. (A) A549 cells were treated with shikonin $(5 \mu \mathrm{M})$, gefitinib $(15 \mu \mathrm{M})$ or the combination for $48 \mathrm{~h}$, and then cells were incubated with PI (upper panel) or JC-1 (lower panel) and analyzed by flow cytometry. (B) Shikonin promoted gefitinib-induced apoptosis in A549, NCI-H1299 and NCI-H460 cells. (C) Shikonin promoted mitochondrial membrane potential induced by gefitinib in three NSCLC cells. "* P<0.01 and ${ }^{* * *} \mathrm{P}<0.001$. PI, propidium iodide.

plus erlotinib group $=3.40$ vs. mean $\mathrm{RTV}_{\text {control group }}=11.58, \mathrm{P}<0.01$; mean RTV ${ }_{\text {shikonin plus gefitinib group }}=4.88$ vs. mean $\mathrm{RTV}_{\text {control group }}=11.58$, $\mathrm{P}<0.05$ ] (Fig. 6). Thus, shikonin sensitized the anti-NSCLC effects of erlotinib/gefitinib on A549 xenografts in vivo.

\section{Discussion}

The first generation EGFR-TKIs, including erlotinib and gefitinib, are used as the first-line treatment of metastatic NSCLC with EGFR exon 19 deletions or exon 21 (L858R) (20,21); however, patients with NSCLC and wild-type EGFR are primarily resistant to erlotinib and gefitinib (6). The T790M mutation in EGFR kinase is the most common $(\sim 50 \%)$ acquired resistance mechanism to first- and second-generation EGFR-TKIs (22). The third generation EGFR-TKIs (rociletinib, osimertinib, HM61713/BI1482694, ASP8273 and EGF816) can overcome EGFR-TKIs resistance mediated by EGFR T790M (23). However, there are numerous mechanisms underlying non-T790M resistance against EGFR-TKIs, including mesenchymal-epithelial transition factor receptor tyrosine kinase (MET) gene amplification, erb $\mathrm{B} 2$ receptor tyrosine kinase gene amplification, reactivation of mitogen-activated protein kinase kinase (MEK-extracellular signal-regulated kinase (ERK) or phosphoinositide 3-kinase (PI3K)-protein kinase B (AKT) signaling pathways (24). Investigating novel combinations of treatment regimens is one of main therapeutic strategies to overcome EGFR-TKI-associated resistance, including primary and acquired resistance, and prolong survival durations for patients with NSCLC. Shikonin may inhibit numerous molecular markers associated with EGFR-TKI resistance, including those involved in the MET-ERK and PI3K-AKT signaling pathways $(25,26)$. In addition, shikonin may be a sensitizer for erlotinib/gefitinib in vitro $(15,16)$. In 
A

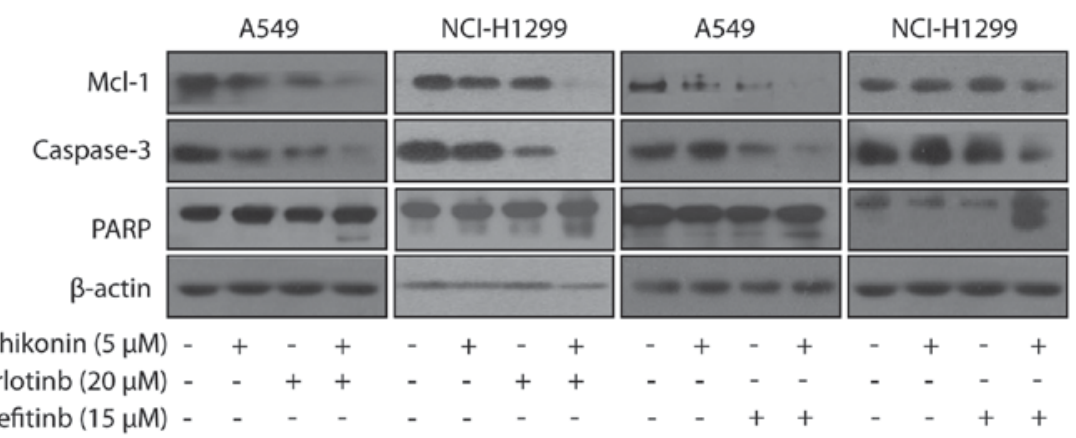

B
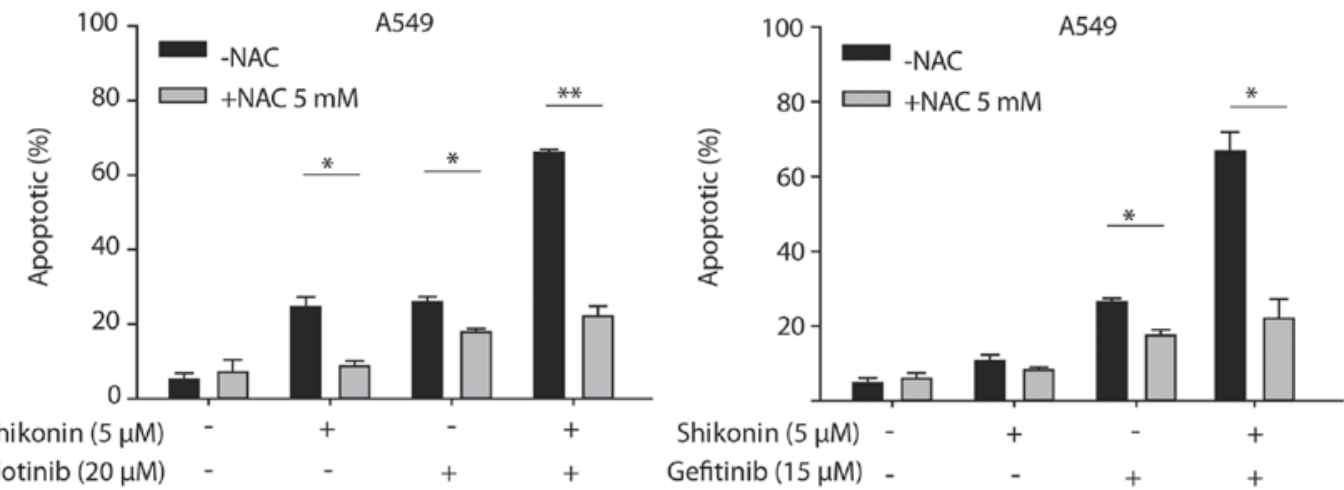

Erlotinib $(20 \mu \mathrm{M})$

Shikonin $(5 \mu \mathrm{M})$ Gefitinib $(15 \mu \mathrm{M})$.

C

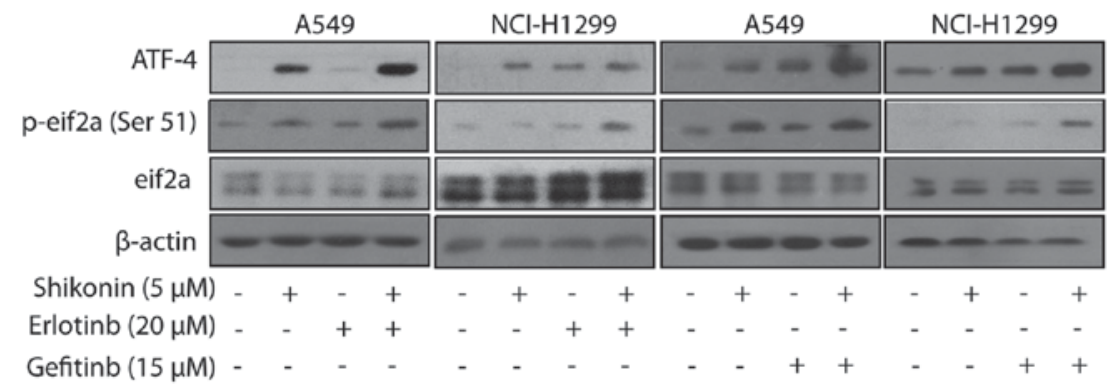

D

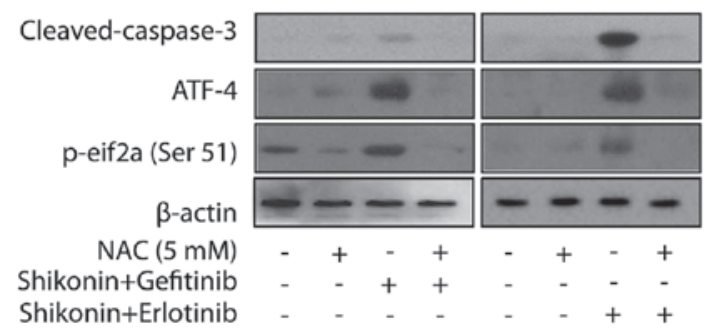

Figure 5. ER stress and ROS are involved in shikonin plus erlotinib/gefitinib treatment-induced apoptosis. (A) A549/NCI-H1299 cells were treated with compounds at indicated concentrations for $48 \mathrm{~h}$, after which the expression of proteins were detected by western blotting. (B) A549 cells were pre-treated with $\mathrm{NAC}(5 \mathrm{mM})$ for $1 \mathrm{~h}$, and then treated with $5 \mu \mathrm{M}$ shikonin and/or $20 \mu \mathrm{M}$ erlotinib (left panel), $5 \mu \mathrm{M}$ shikonin and/or $15 \mu \mathrm{M}$ gefitinib (right panel) for $48 \mathrm{~h}$. PI staining was used to detect apoptosis. (C) Cells were treated with compounds at indicated concentrations for $24 \mathrm{~h}$, after which the expression of proteins were detected by western blotting. (D) A549 cells were pre-treated with NAC $(5 \mathrm{mM})$ for $1 \mathrm{~h}$ and then treated with shikonin+erlotinib or shikonin+gefitinib for $24 \mathrm{~h}$, after which the expression of proteins were detected by western blotting. ${ }^{*} \mathrm{P}<0.05$ and ${ }^{* *} \mathrm{P}<0.01$. Mcl-1, myeloid leukemia cell differentiation protein 1 ; PARP, poly (ADP-ribose) polymerase; ER, endoplasmic reticulum; ROS, reactive oxygen species; NAC, N-acetylcysteine; ATF-4, activating transcription factor 4; PI, propidium iodide; p-eIf $2 \alpha$, phosphorylated-eukaryotic initiation factor $2 \alpha$.

the present study, shikonin was reported to potentiate the anticancer effects of erlotinib/gefitinib on wild-type EGFR NSCLC cells resistant to erlotinib and gefitinib both in vitro and in vivo; however, whether shikonin may sensitize other EGFR-TKIs requires further investigation. Additionally, the dual targeting of EGFR with cetuximab and EGFR-TKIs may be an effective strategy to overcome T790M-mediated drug resistance (27). As shikonin can induce EGFR proteasomal degradation, further investigation is needed to evaluate the anticancer efficiency of shikonin plus EGFR-TKIs on NSCLC cells with EGFR T790M.

Heterogeneous nuclear ribonucleoprotein A1 (hnRNPA1), an abundant ubiquitous nuclear protein, has numerous functions associated with RNA metabolism (28). Shikonin can induce cell death via direct binding-interference with hnRNPA1 (29). HnRNPA1 binds to the 5'untranslated 

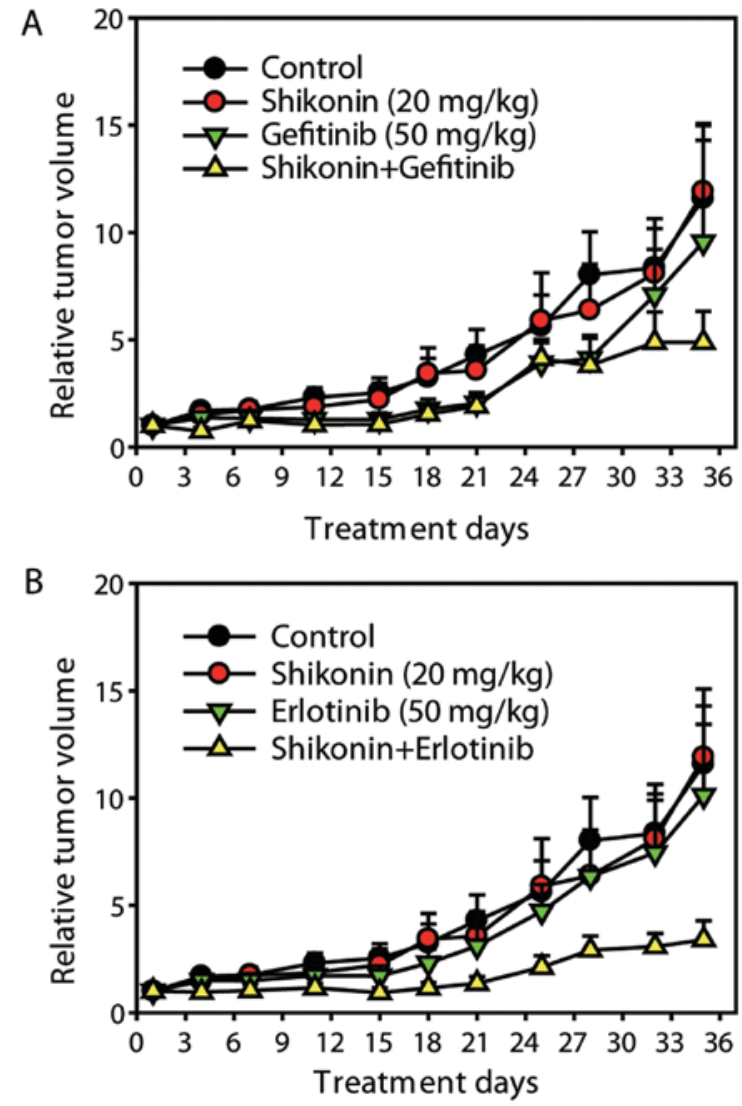

Figure 6. Shikonin sensitizes the anti-NSCLC effects of erlotinib/gefitinib in vivo. (A) The mice with A549 xenografts were randomized to four groups ( $\mathrm{n}=6$ per group) and treated with shikonin $(20 \mathrm{mg} / \mathrm{kg})$, erlotinib $(50 \mathrm{mg} / \mathrm{kg})$, combination, or vehicle for 35 days. RTVs were showed as mean \pm SD (B) The mice with A549 xenografts were randomized to four groups ( $\mathrm{n}=6$ per group) and treated with shikonin ( $20 \mathrm{mg} / \mathrm{kg}$ ), gefitinib (50 mg/kg), combination, or vehicle for 35 days. RTVs were showed as mean \pm SD. NSCLC, non-small cell lung cancer; RTV, Relative Tumor Volume.

region of the mRNA encoding the RON receptor tyrosine kinase and regulates its expression; RON is a member of the c-MET receptor tyrosine kinase family, which has been reported to correlate with EGFR-TKI-associated resistance and poor clinical outcome (30). In addition, co-targeting EGFR and c-MET has been reported to efficiently overcome EGFR-TKI resistance in NSCLC cells (31). Thus, the present study hypothesized that shikonin may suppress MET via hnRNPA1 inhibition and sensitive the anticancer activity of EGFR-TKI, however, further research is required to test this hypothesis.

ER stress induces the unfolded protein response (UPR), which is initiated by the activation of three main stress sensors, including inositol-requiring protein-1 $\alpha$, PERK protein kinase RNA-like ER kinase, and ATF6 (32). ER stress serves a dual role in determining cell survival or cell death. The UPR is primarily a pro-survival process; sustained and/or prolonged stress may result in the induction of cell death (33). ER stress can promote the survival of cancer cells that are tolerant to EGFR-TKIs and has been associated with the pro-survival process (34). In addition, increased levels of intracellular ROS can also induce ER stress and UPR activation, and ROS-mediated ER stress can induce mitochondria-mediated apoptosis via PERK (35-37).
Shikonin induces the apoptosis of prostate cancer cells via the induction of ROS production and ER stress (12). In the present study, it was reported that shikonin may induce apoptosis via ROS-mediated ER stress in wild-type EGFR NSCLC cells. Furthermore, erlotinib/gefitinib can also induce ER stress; shikonin plus erlotinib/gefitinib was more effective in activating ER stress than either agent alone. These findings indicated that ROS-mediated ER stress may be associated with the enhanced mitochondrial apoptosis induced by shikonin plus erlotinib/gefitinib, and that shikonin may promote the transition of cytoprotective ER stress-inducing EGFR-TKI tolerance to apoptotic-promoting ER stress. However, further investigation is required to determine the molecular mechanism underlying the transition between cytoprotective ER stress and apoptosis-promoting ER stress induced by shikonin plus erlotinib/gefitinib.

In summary, the findings of the present study demonstrated that shikonin could sensitize the anticancer activity of erlotinib/gefitinib in wild-type EGFR NSCLC cells both in vitro and in vivo. Furthermore, ROS-mediated ER stress may be associated with the enhanced anti-NSCLC effects induced by shikonin plus erlotinib/gefitinib. The present study suggested that combining shikonin with EGFR-TKIs may be a novel theray for wild-type EGFR NSCLC and may also provide insight into the application of shikonin in other clinical research fields.

\section{Acknowledgements}

Not applicable.

\section{Funding}

The present study was funded by Zhejiang Province Medical Key Discipline Construction (grant no. 2018-2-03), National natural science foundation of china (grant no. 81702887), Hangzhou major science and technology project (grant no. 20172016A01), Fund of Hangzhou medical key discipline construction (grant no. 2017-51-07), Zhejiang provincial foundation of natural science (grant no. LQ16H310004), Public-service technology research plan of Zhejiang province (grant nos. 2015C33269 and 2016C33210), High-level talents coming back from abroad innovation and entrepreneurship program in Hangzhou (grant no. 2051), Zhejiang provincial program for the cultivation of high-level innovative health talents (grant no. 2010-190-4), Scientific and technological developing scheme of Hangzhou city (grant nos. 20150733Q14 and 20140633B03).

\section{Availability of data and materials}

The analyzed data sets generated during the study are available from the corresponding author on reasonable request.

\section{Authors' contributions}

Y-LL, XH, Q-YL, FW, BZ, KD and B-QT performed the experiments. N-ML and CZ conceived and designed the study. $\mathrm{CZ}$ wrote the manuscript. All authors read and approved the manuscript. 


\section{Ethics approval and consent to participate}

All animal manipulations were performed in accordance with the National institutes of health guide for the care and use of laboratory animals, and were approved by the Zhejiang University City College animal care and use committee. This article does not contain any studies with human participants performed by any of the authors.

\section{Patient consent for publication}

Not applicable.

\section{Competing interests}

The authors declare that they have no competing interests.

\section{References}

1. Davies J, Patel M, Gridelli C, de Marinis F, Waterkamp D and McCusker ME: Real-world treatment patterns for patients receiving second-line and third-line treatment for advanced non-small cell lung cancer: A systematic review of recently published studies. PLoS One 12: e0175679, 2017.

2. Chen Z, Fillmore CM, Hammerman PS, Kim CF and Wong KK: Non-small-cell lung cancers: A heterogeneous set of diseases. Nat Rev Cancer 14: 535-546, 2014.

3. Jiang T, Qiao M, Zhou F, Ren S, Su C and Zhou C: Effect of combined therapy inhibiting EGFR and VEGFR pathways in non-small-cell lung cancer on progression-free and overall survival. Clin Lung Cancer 18: 421-431.e3, 2017.

4. Takeda $M$ and Nakagawa $K$ : Toxicity profile of epidermal growth factor receptor tyrosine kinase inhibitors in patients with epidermal growth factor receptor gene mutation-positive lung cancer. Mol Clin Oncol 6: 3-6, 2017.

5. Han W and Du Y: Recent development of the second and third generation irreversible epidermal growth factor receptor inhibitors. Chem Biodivers 14, 2017.

6. Paez JG, Jänne PA, Lee JC, Tracy S, Greulich H, Gabriel S, Herman P, Kaye FJ, Lindeman N, Boggon TJ, et al: EGFR mutations in lung cancer: Correlation with clinical response to gefitinib therapy. Science 304: 1497-1500, 2004.

7. Zhu CQ, da Cunha Santos G, Ding K, Sakurada A, Cutz JC, Liu N, Zhang T, Marrano P, Whitehead M, Squire JA, et al: Role of KRAS and EGFR as biomarkers of response to erlotinib in national cancer institute of canada clinical trials group study BR.21. J Clin Oncol 26: 4268-4275, 2008.

8. Jeung YJ, Kim HG, Ahn J, Lee HJ, Lee SB, Won M, Jung CR, Im JY, Kim BK, Park SK, et al: Shikonin induces apoptosis of lung cancer cells via activation of FOXO3a/EGR1/SIRT1 signaling antagonized by p300. Biochim Biophys Acta 1863: 2584-2593, 2016.

9. Zhou Z, Lu B, Wang C, Wang Z, Luo T, Piao M, Meng F, Chi G, Luo Y and Ge P: RIP1 and RIP3 contribute to shikonin-induced DNA double-strand breaks in glioma cells via increase of intracellular reactive oxygen species. Cancer Lett 390: 77-90, 2017.

10. Komi Y, Suzuki Y, Shimamura M, Kajimoto S, Nakajo S, Masuda M, Shibuya M, Itabe H, Shimokado K, Oettgen P, et al: Mechanism of inhibition of tumor angiogenesis by beta-hydroxyisovalerylshikonin. Cancer Sci 100: 269-277, 2009.

11. Liang W, Cai A, Chen G, Xi H, Wu X, Cui J, Zhang K, Zhao X, Yu J, Wei B and Chen L: Shikonin induces mitochondria-mediated apoptosis and enhances chemotherapeutic sensitivity of gastric cancer through reactive oxygen species. Sci Rep 6: 38267, 2016.

12. Gara RK, Srivastava VK, Duggal S, Bagga JK, Bhatt M, Sanyal S and Mishra DP: Shikonin selectively induces apoptosis in human prostate cancer cells through the endoplasmic reticulum stress and mitochondrial apoptotic pathway. J Biomed Sci 22: 26, 2015.

13. Li W, Liu J, Jackson K, Shi R and Zhao Y: Sensitizing the therapeutic efficacy of taxol with shikonin in human breast cancer cells. PLoS One 9: e94079, 2014.
14. Song J, Zhao Z, Fan X, Chen M, Cheng X, Zhang D, Wu F, Ying $X$ and Ji J: Shikonin potentiates the effect of arsenic trioxide against human hepatocellular carcinoma in vitro and in vivo. Oncotarget 7: 70504-70515, 2016.

15. Zhao Q, Kretschmer N, Bauer R and Efferth T: Shikonin and its derivatives inhibit the epidermal growth factor receptor signaling and synergistically kill glioblastoma cells in combination with erlotinib. Int J Cancer 137: 1446-1456, 2015.

16. Li X, Fan XX, Jiang ZB, Loo WT, Yao XJ, Leung EL, Chow LW and Liu L: Shikonin inhibits gefitinib-resistant non-small cell lung cancer by inhibiting TrxR and activating the EGFR proteasomal degradation pathway. Pharmacol Res 115: 45-55, 2017.

17. Li YL, Sun J, Hu X, Pan YN, Yan W, Li QY, Wang F, Lin NM and Zhang C: Epothilone B induces apoptosis and enhances apoptotic effects of ABT-737 on human cancer cells via PI3K/AKT/mTOR pathway. J Cancer Res Clin Oncol 142: 2281-2289, 2016.

18. Zhang C, Shi J, Mao SY, Xu YS, Zhang D, Feng LY, Zhang B, Yan YY, Wang SC, Pan JP, et al: Role of p38 MAPK in enhanced human cancer cells killing by the combination of aspirin and ABT-737. J Cell Mol Med 19: 408-417, 2015.

19. Chou TC and Talalay P: Quantitative analysis of dose-effect relationships: The combined effects of multiple drugs or enzyme inhibitors. Adv Enzyme Regul 22: 27-55, 1984.

20. Niu FY and Wu YL: Novel agents and strategies for overcoming EGFR TKIs resistance. Exp Hematol Oncol 3: 2, 2014.

21. Engelman JA and Jänne PA: Mechanisms of acquired resistance to epidermal growth factor receptor tyrosine kinase inhibitors in non-small cell lung cancer. Clin Cancer Res 14: 2895-2899, 2008.

22. Li YL, Pan YN, Wu WJ, Mao SY, Sun J, Zhao YM, Dong JY, Zhang DY, Pan JP, Zhang C and Lin NM: Evodiamine induces apoptosis and enhances apoptotic effects of erlotinib in wild-type EGFR NSCLC cells via S6K1-mediated Mcl-1 inhibition. Med Oncol 33: 16, 2016.

23. Oxnard GR, Thress KS, Alden RS, Lawrance R, Paweletz CP, Cantarini M, Yang JC, Barrett JC and Jänne PA: Association between plasma genotyping and outcomes of treatment with osimertinib (AZD9291) in advanced non-small-cell lung cancer. J Clin Oncol 34: 3375-3382, 2016.

24. Suda K, Rivard CJ, Mitsudomi T and Hirsch FR: Overcoming resistance to EGFR tyrosine kinase inhibitors in lung cancer, focusing on non-T790M mechanisms. Expert Rev Anticancer Ther 17: 779-786, 2017.

25. Hsieh YS, Liao CH, Chen WS, Pai JT and Weng MS: Shikonin inhibited migration and invasion of human lung cancer cells via suppression of c-Met-mediated epithelial-to-mesenchymal transition. J Cell Biochem 118: 4639-4651, 2017.

26. Gwon SY, Ahn JY, Jung CH, Moon BK and Ha TY: Shikonin suppresses ERK $1 / 2$ phosphorylation during the early stages of adipocyte differentiation in 3T3-L1 cells. BMC Complement Altern Med 13: 207, 2013.

27. Regales L, Gong Y, Shen R, de Stanchina E, Vivanco I, Goel A, Koutcher JA, Spassova M, Ouerfelli O, Mellinghoff IK, et al: Dual targeting of EGFR can overcome a major drug resistance mutation in mouse models of EGFR mutant lung cancer. J Clin Invest 119: 3000-3010, 2009.

28. Jean-Philippe J, Paz S and Caputi M: hnRNP A1: The Swiss army knife of gene expression. Int J Mol Sci 14: 18999-19024, 2013.

29. Yin SY, Efferth T, Jian FY, Chen YH, Liu CI, Wang AH, Chen YR, Hsiao PW and Yang NS: Immunogenicity of mammary tumor cells can be induced by shikonin via direct binding-interference with hnRNPA1. Oncotarget 7: 43629-43653, 2016.

30. Cammas A, Lacroix-Triki M, Pierredon S, Le Bras M, Iacovoni JS, Teulade-Fichou MP, Favre G, Roché H, Filleron T, Millevoi S and Vagner S: hnRNP A1-mediated translational regulation of the G quadruplex-containing RON receptor tyrosine kinase mRNA linked to tumor progression. Oncotarget 7: 16793-16805, 2016.

31. Wu DW, Chen TC, Huang HS and Lee H: TC-N19, a novel dual inhibitor of EGFR and cMET, efficiently overcomes EGFR-TKI resistance in non-small-cell lung cancer cells. Cell Death Dis 7: e2290, 2016.

32. Sano R and Reed JC: ER stress-induced cell death mechanisms. Biochim Biophys Acta 1833: 3460-3470, 2013.

33. Corazzari M, Gagliardi M, Fimia GM and Piacentini M: Endoplasmic reticulum stress, unfolded protein response, and cancer cell fate. Front Oncol 7: 78, 2017. 
34. Terai H, Kitajima S, Potter DS, Matsui Y, Quiceno LG, Chen T, Kim TJ, Rusan M, Thai TC, Piccioni F, et al: ER stress signaling promotes the survival of cancer 'persister cells' tolerant to EGFR tyrosine kinase inhibitors. Cancer Res 78: 1044-1057, 2018.

35. Malhotra JD and Kaufman RJ: Endoplasmic reticulum stress and oxidative stress: A vicious cycle or a double-edged sword? Antioxid Redox Signal 9: 2277-2293, 2007.

36. Rutkowski DT and Kaufman RJ: That which does not kill me makes me stronger: Adapting to chronic ER stress. Trends Biochem Sci 32: 469-476, 2007.
37. Verfaillie T, Rubio N, Garg AD, Bultynck G, Rizzuto R, Decuypere JP, Piette J, Linehan C, Gupta S, Samali A and Agostinis P: PERK is required at the ER-mitochondrial contact sites to convey apoptosis after ROS-based ER stress. Cell Death Differ 19: 1880-1891, 2012.

cc (7) (9) This work is licensed under a Creative Commons cc) $\mathrm{EY}$ NO NO Attribution-NonCommercial-NoDerivatives 4.0 International (CC BY-NC-ND 4.0) License. 